The Astronomical Journal, 130:1784-1794, 2005 October

(C) 2005. The American Astronomical Society. All rights reserved. Printed in U.S.A.

\title{
THE MULTITUDE OF MOLECULAR HYDROGEN KNOTS IN THE HELIX NEBULA ${ }^{1}$
}

\author{
Margaret Meixner and Peter McCullough \\ Space Telescope Science Institute, 3700 San Martin Drive, Baltimore, MD 21218; meixner@stsci.edu, pmcc@stsci.edu \\ Joel Hartman \\ Harvard-Smithsonian Center for Astrophysics, 60 Garden Street, Cambridge, MA 02138; jhartman@cfa.harvard.edu \\ MinHo Son \\ Department of Physics and Astronomy, Charles and 34th Street, Bloomberg Center, Johns Hopkins University, \\ Baltimore, MD 21218; mhson@pha.jhu.edu \\ AND \\ Angela SPECK \\ Department of Physics, University of Missouri, Columbia, MO 65211; speckan@missouri.edu \\ Received 2005 March 25; accepted 2005 June 14
}

\begin{abstract}
We present Hubble Space Telescope NICMOS imaging of the $\mathrm{H}_{2} 2.12 \mu \mathrm{m}$ emission in five fields in the Helix Nebula ranging in radial distance from $250^{\prime \prime}$ to $450^{\prime \prime}$ from the central star. The images reveal arcuate structures with their apexes pointing toward the central star. These molecular hydrogen knots are most highly structured in the fields closest to the central star and become increasingly less structured with increasing radius. Comparison of these images with ground-based images of comparable resolution reveals that the molecular gas is more highly clumped than the ionized gas line tracers. From our images, we determine an average number density of knots in the molecular gas ranging from 162 knots $\operatorname{arcmin}^{-2}$ in the denser regions to 18 knots $\operatorname{arcmin}^{-2}$ in the lower density outer regions. The decreasing number density of $\mathrm{H}_{2}$ knots in the outer regions creates a lower filling factor of neutral and molecular gas emission in the radio observations of $\mathrm{CO}$ and $\mathrm{H}$ I and may explain why these outer regions, where we clearly detect $\mathrm{H}_{2} 2.12 \mu \mathrm{m}$, fall below the detection limit of the radio observations. Using this new number density, we estimate the total number of knots in the Helix to be $\sim 23,000$, which is a factor of 6.5 larger than previous estimates. The total neutral gas mass in the Helix is $0.35 M_{\odot}$ assuming a mass of $\sim 1.5 \times 10^{-5} M_{\odot}$ for the individual knots. The $\mathrm{H}_{2}$ emission structure of the entire Helix Nebula supports the recent interpretation of the Helix as a nearly pole-on polypolar planetary nebula (PN). The $\mathrm{H}_{2}$ intensity, (5-9) $\times 10^{-5} \mathrm{ergs} \mathrm{s}^{-1} \mathrm{~cm}^{-2} \mathrm{sr}^{-1}$, remains relatively constant with projected distance from the central star, suggesting a heating mechanism for the molecular gas that is distributed almost uniformly in the knots throughout the nebula. The temperature and $\mathrm{H}_{2} 2.12 \mu \mathrm{m}$ intensity of the knots can be approximately explained by photodissociation regions (PDRs) in the individual knots; however, theoretical PDR models of PNs underpredict the intensities of some knots by a factor of 10 . The brightest $\mathrm{H}_{2}$ emission $\left(\sim 3 \times 10^{-4} \mathrm{ergs} \mathrm{s}^{-1} \mathrm{~cm}^{-2} \mathrm{sr}^{-1}\right)$ may be enhanced by a larger than unity area filling factor of $\mathrm{H}_{2}$ knots or may be an individual $\mathrm{H}_{2}$ knot exposed to direct starlight, causing rapid photoevaporation compared with the more embedded knots of the disk.
\end{abstract}

Key word: planetary nebulae: individual (NGC 7293)

\section{INTRODUCTION}

Approximately 50 planetary nebulae (PNs) are currently known to have "small-scale" heterogeneities located inside or outside the main ionized nebulae (Gonçalves et al. 2001). Cometary knots are a subcategory of small-scale structures found commonly in nearby, evolved PNs (O'Dell et al. 2002). Because of its close proximity (213 pc; Harris et al. 1997), the Helix Nebula (NGC 7293) is the best case to study the structure and excitation conditions of cometary knots. The nature of the cometary knots in the Helix was first established by Meaburn et al. (1992). The detailed structure of the cometary knots has been resolved in ionized gas lines in the optical by O'Dell \& Handron (1996), with further detailed analysis by Burkert \&

\footnotetext{
${ }^{1}$ Based in part on observations with the NASA/ESA Hubble Space Telescope obtained at the Space Telescope Science Institute, which is operated by the Association of Universities for Research in Astronomy, Inc., under NASA contract NAS5-26555.
}

O'Dell (1998) and O'Dell et al. (2000). The emerging optical picture of the cometary knots reveals that they are neutral gas condensations that appear as comet-like structures with rims bright in $\mathrm{H} \alpha$ and tails that appear as shadows in [O III] and that point away from the central star. The rim of low-excitation ionized gas has a steep temperature gradient, indicating that the knots are photoevaporating and that ionization fronts are advancing into the knots (O'Dell et al. 2000). A recent analysis of knots over the whole Helix Nebula by O'Dell et al. (2004) revealed a new three-dimensional picture for the main ring of the Helix: it is composed of a disk structure and an outer ring tilted almost perpendicularly with respect to the disk. Within each of these components they observed a similar, progressive evolution in the structure of the knots. The knots closest to the central star and clearly inside of the ionization front appeared crisply carved with the brightest rims. The knots furthest from the central star appeared slightly more amorphous in their structure, with less well defined rims. These optical observations appear to support the theory that these knots were initially 


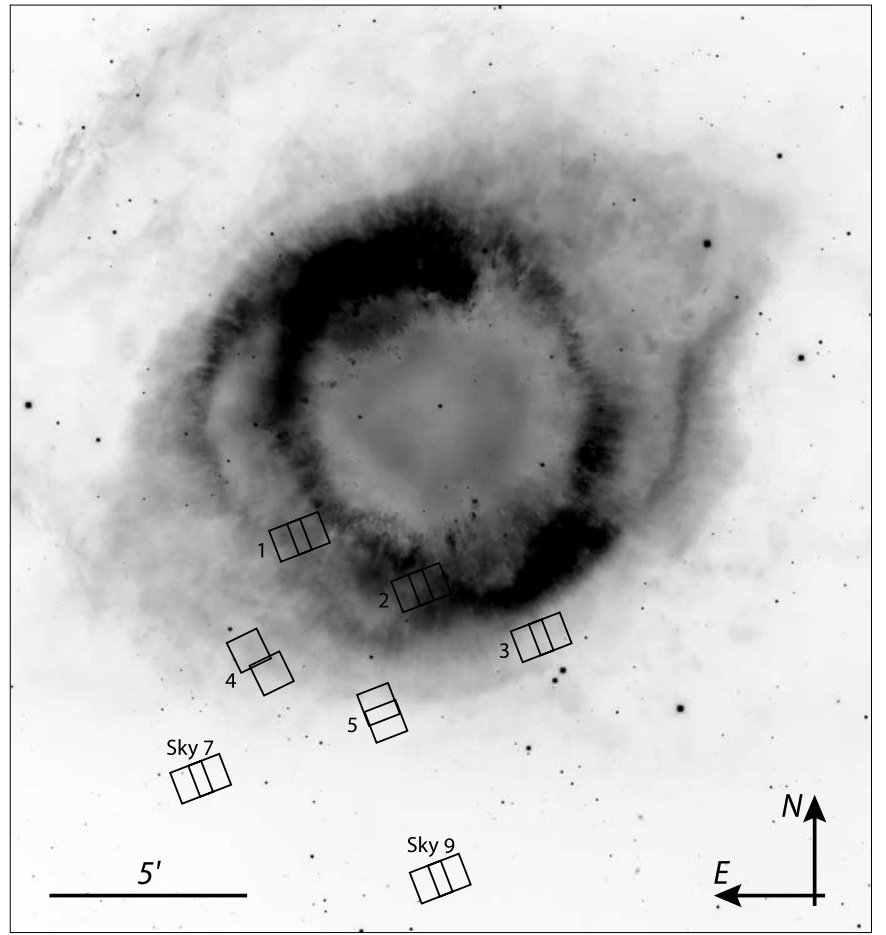

Fig. 1.-Negative picture of the Helix from O'Dell et al. (2004) showing the optical structure on a large scale. The NICMOS field positions are overlaid as black rectangles.

formed earlier by instabilities at the ionization front, or perhaps by the interaction of the fast stellar wind, and sculpted by interaction with the harsh radiation field of the central star (Capriotti 1973).

In contrast to the high angular resolution $\left(\sim 0^{\prime \prime} 01\right)$ optical studies of the ionized gas lines in the cometary knots, molecular gas observations have had lower angular resolution $\left(4^{\prime \prime}-\right.$ $\left.41^{\prime \prime}\right)$ and sensitivity, making it difficult to determine the detailed structure and excitation of the main gas component of the cometary knots. These low-resolution studies have revealed that the Helix has retained a significant amount of molecular gas (Young et al. 1999; Huggins \& Healy 1986; Speck et al. 2002; Kastner et al. 1994) and that the molecular gas appears to be very clumpy and is probably confined to the cometary knot structures (Speck et al. 2002; Huggins et al. 2002). The only detailed study of an isolated cometary knot that is close to the central star shows no evidence for large velocities in the molecular gas, ruling out a stellar wind shaping the knot, and reveals the stratified structure for the ionized and molecular gas emissions that is expected in a photodissociation region (PDR;
Huggins et al. 2002). However, since recent optical studies show an evolution of the knot structure with radial distance from the central star (O'Dell et al. 2004), it is not clear that this single knot study is representative of all the knots in the nebula. In order to determine the structure and excitation of the $\mathrm{H}_{2}$ emission in the cometary knots at comparable resolution to optical images across the Helix, we pursued high angular resolution $\left(\sim 0^{\prime \prime} 2\right)$ NICMOS NIC3 F212 $\mathrm{N} \mathrm{H}_{2}$ images at several locations in the nebula, in parallel with the Hubble Space Telescope (HST) ACS program recently published by O'Dell et al. (2004).

The remainder of this paper is organized as follows. In $\S 2$ we report the observation and data-processing procedures. In $\S 3$ we discuss the major observational results and how these relate to the optical ionized gas line emissions imaged by O'Dell et al. (2004). In $\S 4$ we interpret the observations in the context of the current understanding of Helix's three-dimensional structure and discuss the number density, mass, evolution, and excitation of the knots as revealed by our $\mathrm{H}_{2}$ images. We summarize our conclusions in $\S 5$.

\section{OBSERVATIONS}

The Hubble Helix project (GO program 9700; principal investigator: M. Meixner) imaged the Helix Nebula during the 2002 Leonids meteor shower, which presented a risk to the HST. The imaging involved a nine-panel mosaic of the Helix using the ACS WFC instrument in the F658N filter (transmitting equally well both the $\mathrm{H} \alpha \lambda 6563$ and [ $\mathrm{N}$ II] $\lambda 6584$ lines) and the F502N filter (dominated by the [O $\mathrm{III}] 25007$ line). In parallel with the ACS imaging, we used NICMOS (Thompson et al. 1998) to image seven of the possible nine field positions, five of which landed on the nebula (positions 1, 2, 3, 4, and 5) and two of which were off the nebula (positions 7 and 9) and used for background measurements for the five fields on the nebula. Figure 1 shows the location of these fields on the Helix, and the right ascension and declination of the field centers for field positions 1, 2, 3, 4, and 5 are listed in Table 1. These parallel NICMOS field positions had insignificant overlap with the ACS images. Because we wanted maximum field of view (FOV) and our target was a diffuse nebula, we used the NIC3 camera, 0.2 pixel $^{-1}$, with the F212N filter to image the $\mathrm{H}_{2} 2.12 \mu \mathrm{m}$ line emission in the nebula. For field positions 1 and 2, half the time was spent in the $\mathrm{Pa} \alpha$ filter $\mathrm{F} 187 \mathrm{~N}$, which had a sufficiently low signal-to-noise ratio as to be useless and is not discussed further. For each field position, the two dither positions for ACS resulted in two slightly overlapping NICMOS NIC3 images. The NIC3 MULTIACCUM, FAST readout mode was used. The Hubble Helix project and its results (McCullough et al. 2002) immediately went into the public domain and have been briefly discussed by Meixner et al.

TABLE 1

NiCmos Field Position Centers and the $\mathrm{H}_{2}$ Knot Positions with Respect to These Centers

\begin{tabular}{|c|c|c|c|c|}
\hline Positions & $\begin{array}{l}\text { R.A. (J2000.0) } \\
\text { of }(0,0)\end{array}$ & $\begin{array}{l}\text { Decl. }(\mathrm{J} 2000.0) \\
\text { of }(0,0)\end{array}$ & $\begin{array}{l}\Delta \text { R.A. to } \mathrm{H}_{2} \mathrm{Knot} \\
\quad(\operatorname{arcsec})\end{array}$ & $\begin{array}{l}\Delta \text { Decl. to } \mathrm{H}_{2} \mathrm{Knot} \\
\quad(\operatorname{arcsec})\end{array}$ \\
\hline 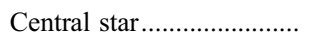 & 222938.5 & -205013.5 & & \\
\hline Position 1 ........................... & 222953.03 & -205327.87 & -28.32 & 31.57 \\
\hline Position $2 \ldots \ldots \ldots \ldots$ & 222940.23 & -205444.07 & -34.51 & 18.68 \\
\hline 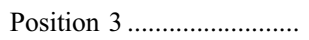 & 222927.44 & -20560.26 & -4.97 & 22.53 \\
\hline 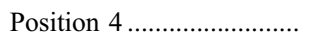 & 222959.67 & -205621.79 & 7.11 & 41.01 \\
\hline Position $5 \ldots \ldots \ldots$ & 222944.98 & -205810.38 & -7.01 & 35.72 \\
\hline
\end{tabular}

NoтE.- Units of right ascension are hours, minutes, and seconds, and units of declination are degrees, arcminutes, and arcseconds. 


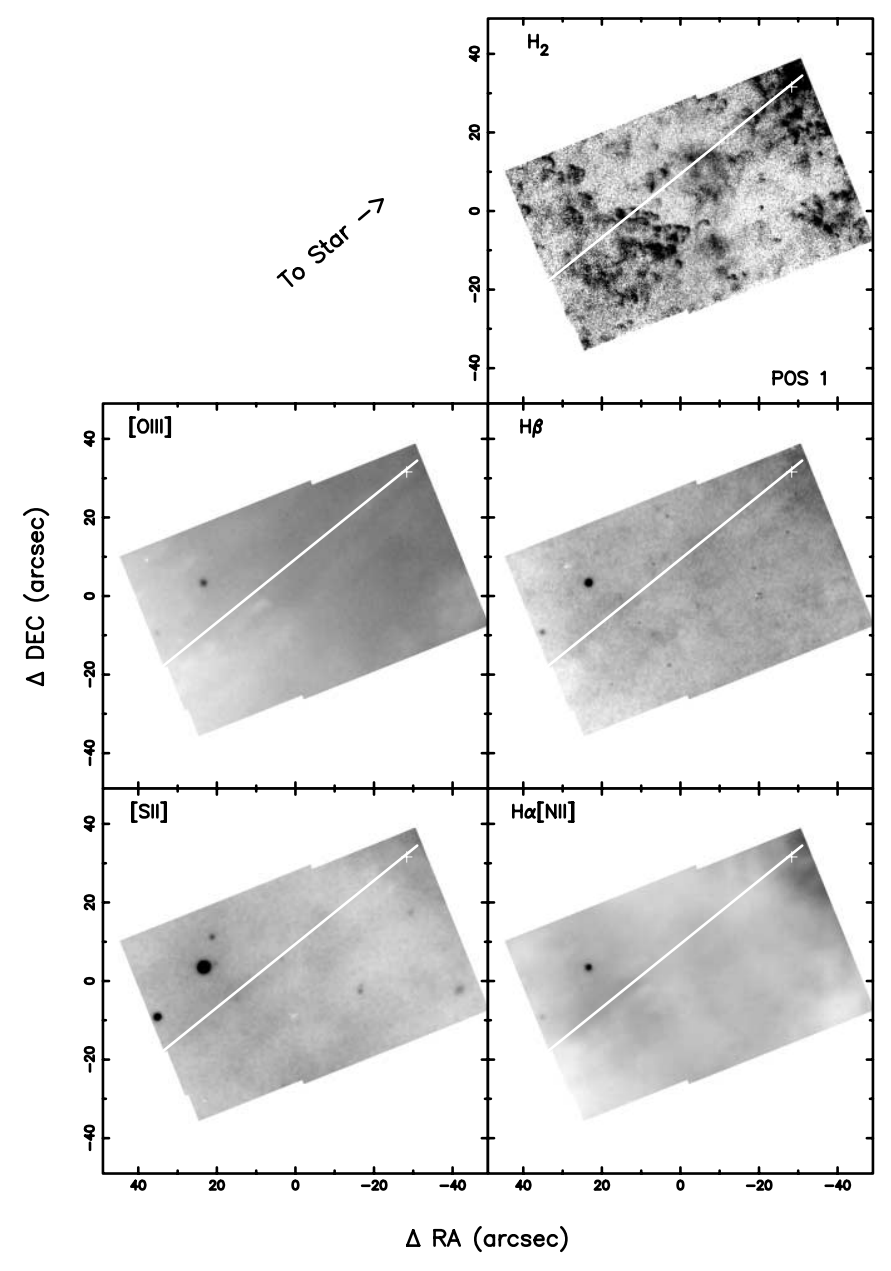

FIG. 2.-NICMOS position 1. The observed $\mathrm{H}_{2} 2.12 \mu \mathrm{m}$ emission is in the top right panel, with the [O III] (middle left), $\mathrm{H} \beta$ (middle right), $\mathrm{H} \alpha+[\mathrm{N}$ II] (bottom right), and $\left[\mathrm{S}_{\mathrm{II}}\right]$ (bottom left) emission from the same region extracted from the CTIO ground-based images of O'Dell et al. (2004). The white cross marks the location where the flux measurements of Table 2 were taken. The white line shows the location of the crosscut shown in Fig. 7. The approximate direction of the central star is shown.

(2004). The ACS images were analyzed in combination with ground-based Cerro Tololo Inter-American Observatory (CTIO) images in similar filters and have been published by O'Dell et al. (2004). In this work we analyze and discuss the NICMOS $\mathrm{H}_{2}$ $2.12 \mu \mathrm{m}$ emission and its relation to the ionized gas at high spatial resolution. The high-level data products for both the ACS images presented by O'Dell et al. (2004) and the NICMOS images presented in this paper are available from the STScI MAST Web site. ${ }^{2}$

The NICMOS NIC3 images were reduced and calibrated using the standard set of NICMOS calibration programs provided in the latest version (ver. 3.1) of IRAF STSDAS. ${ }^{3}$ The CALNICA calibration routines in STSDAS perform zero-read signal correction, bias subtraction, dark subtraction, detector nonlinearity correction, flat-field correction, and flux calibration. The pedestal effect was removed by first manually inserting the STSDAS task biaseq in the middle of the CALNICA processes (before flat-fielding) and then employing the STADAS task

\footnotetext{
${ }^{2}$ See http://archive.stsci.edu/hst/helix/data.html.

${ }^{3}$ STSDAS is a product of the Space Telescope Science Institute, which is operated by the Association of Universities for Research in Astronomy, Inc., for NASA.
}

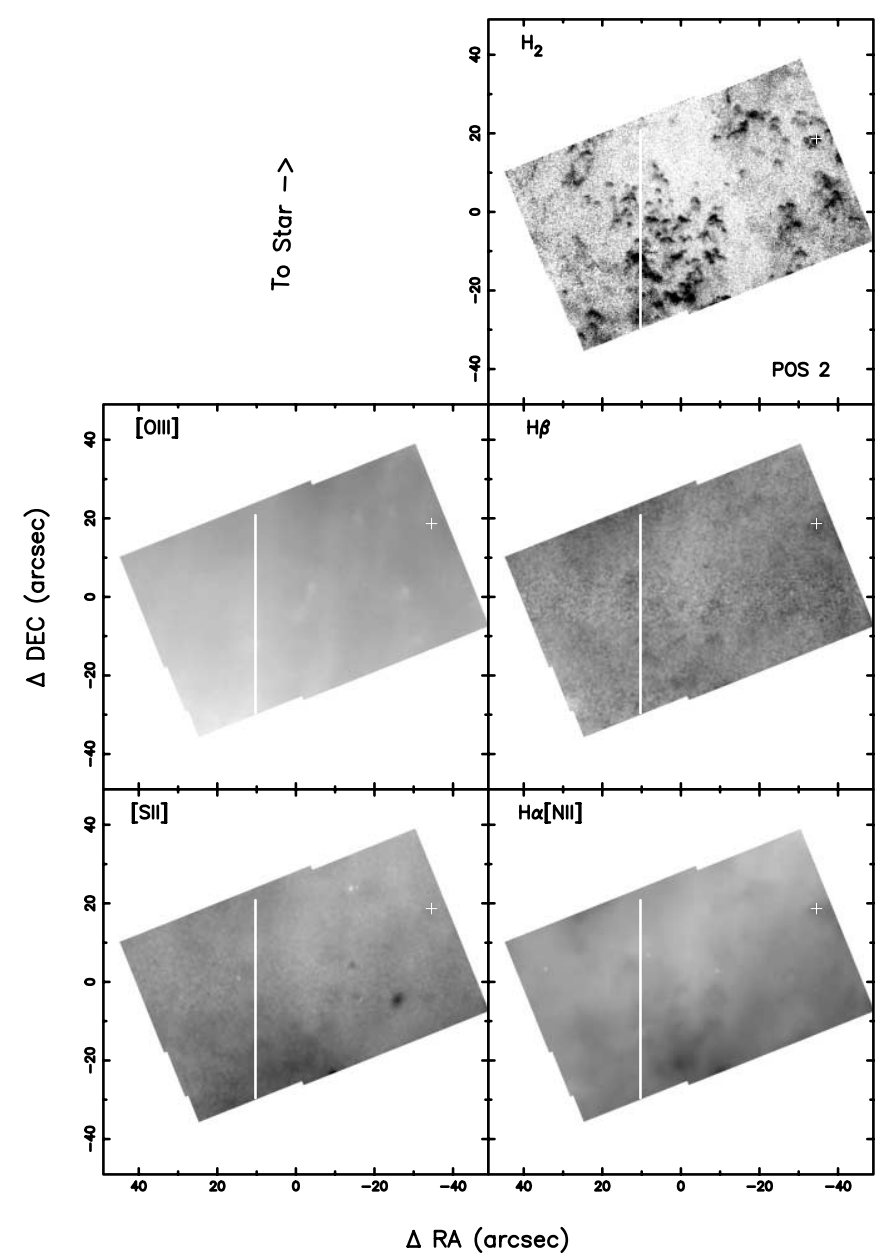

FIG. 3.-Same as Fig. 1 but for NICMOS position 2.

pedsub after the CALNICA processes. Cosmic rays were identified and replaced by the median filtered pixel value.

The four dither positions for field positions 7 and 9 were combined to make a "sky" image that is completely attributed to the telescope emission. This "sky" was subtracted from each of the dither frames for field positions $1-5$, resulting in an $\mathrm{H}_{2}$-dominated emission frame. The continuum emission from the Helix Nebula in the F $212 \mathrm{~N}$ filter is negligible in the sky-subtracted images, as demonstrated by Speck et al. (2002). The two dither positions for each field position were combined using drizzle technique, which magnifies the images by a factor of 2 . The final drizzled images have a plate scale of $0^{\prime \prime} 10$ pixel $^{-1}$ and have been rotated so that north is up and east is to the left. Total integration times for the final, drizzled F212N images ranged from $768 \mathrm{~s}$ for field positions 1 and 2 to $1792 \mathrm{~s}$ for field position 3 . Figures $2-6$ show the final NICMOS images for field positions $1-5$, respectively, in comparison with the optical emission line images from O'Dell et al. (2004). These final NICMOS images, in units of count rate per pixel, are available at the MAST Web site under the Hubble Helix project.

In order to discern the relative distribution of the $\mathrm{H}_{2}$ line emission with the ionized gas line tracers, we compare our results with comparable resolution optical images. The overlap between the NICMOS fields and the ACS fields is insignificant. Fortunately, O'Dell et al. (2004) presented ground-based CTIO images of $\mathrm{H} \alpha+[\mathrm{N} \mathrm{II}]$, [O III], $\mathrm{H} \beta$, and [S II] for the entire Helix Nebula at comparable resolution to our NICMOS images. We registered these CTIO images using the world coordinate system 


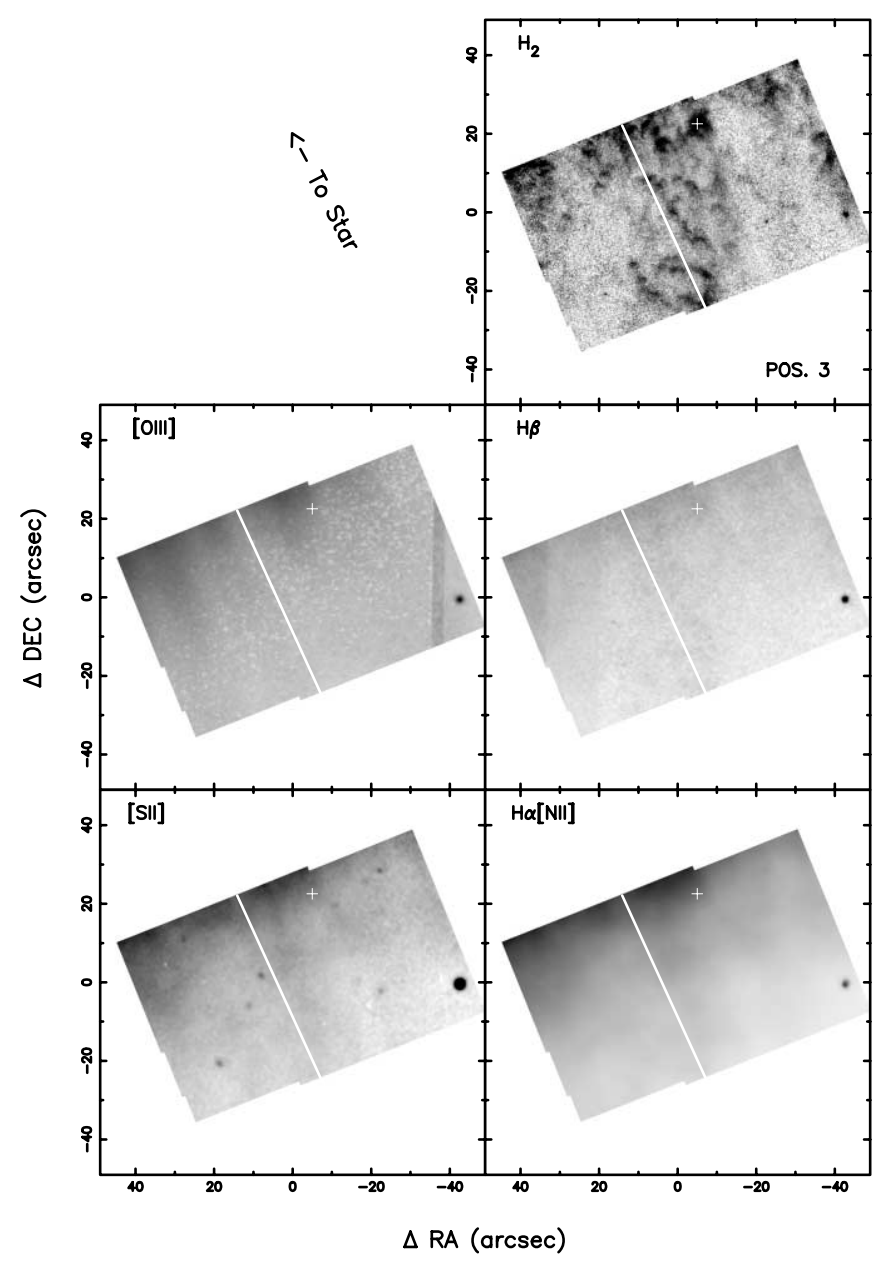

FIg. 4.- Same as Fig. 1 but for NICMOS position 3.

(WCS) of the NICMOS images. The initial comparison, using just the absolute coordinates of the NICMOS and CTIO images, permitted a close enough alignment to identify at least 1 star in common between the NICMOS and CTIO images, which was used for translational alignment. The four CTIO images had 6-7 stars in common with each other, and our detailed comparison revealed small rotational errors between them of up to 0.08 relative angular rotations. Using the star in common with the NICMOS image as the "origin," we improved the relative rotational alignment of the CTIO images to better than 0.005 . The CTIO images were then translated to the NICMOS WCS position by aligning the star in common using the task register in IRAF. Only the parts of the CTIO images that overlap with the NICMOS field positions are shown.

For each field position we selected a prominent $\mathrm{H}_{2}$ knot, labeled with a cross in the figures, for surface brightness measurements of the $\mathrm{H}_{2}$ emission and made a crosscut along the radial direction from the central star in order to quantify the relation between $\mathrm{H}_{2}$ emission and distance from the central star. These positions and crosscuts are labeled on Figures 2-6 and the locations of the $\mathrm{H}_{2}$ knots with respect to the field centers are listed in Table 1. Table 2 lists the surface brightness measurements for $\mathrm{H}_{2}$ and the optical line tracers at the knot position. The average surface brightness of the $\mathrm{H}_{2}$ knot emission (in units of $\mathrm{DN}$ pixel ${ }^{-1}$, where $\mathrm{DN}$ is counts per second) was determined for a circular aperture enclosing the brightest part of the $\mathrm{H}_{2}$ emission $(\sim 3$ pixel radius centered on the cross). We then converted this average into physical units by multiplying by $2.44929 \times 10^{-18} \mathrm{ergs} \mathrm{s}^{-1} \mathrm{~cm}^{-2} \AA^{-1} \mathrm{DN}^{-1}$, which is

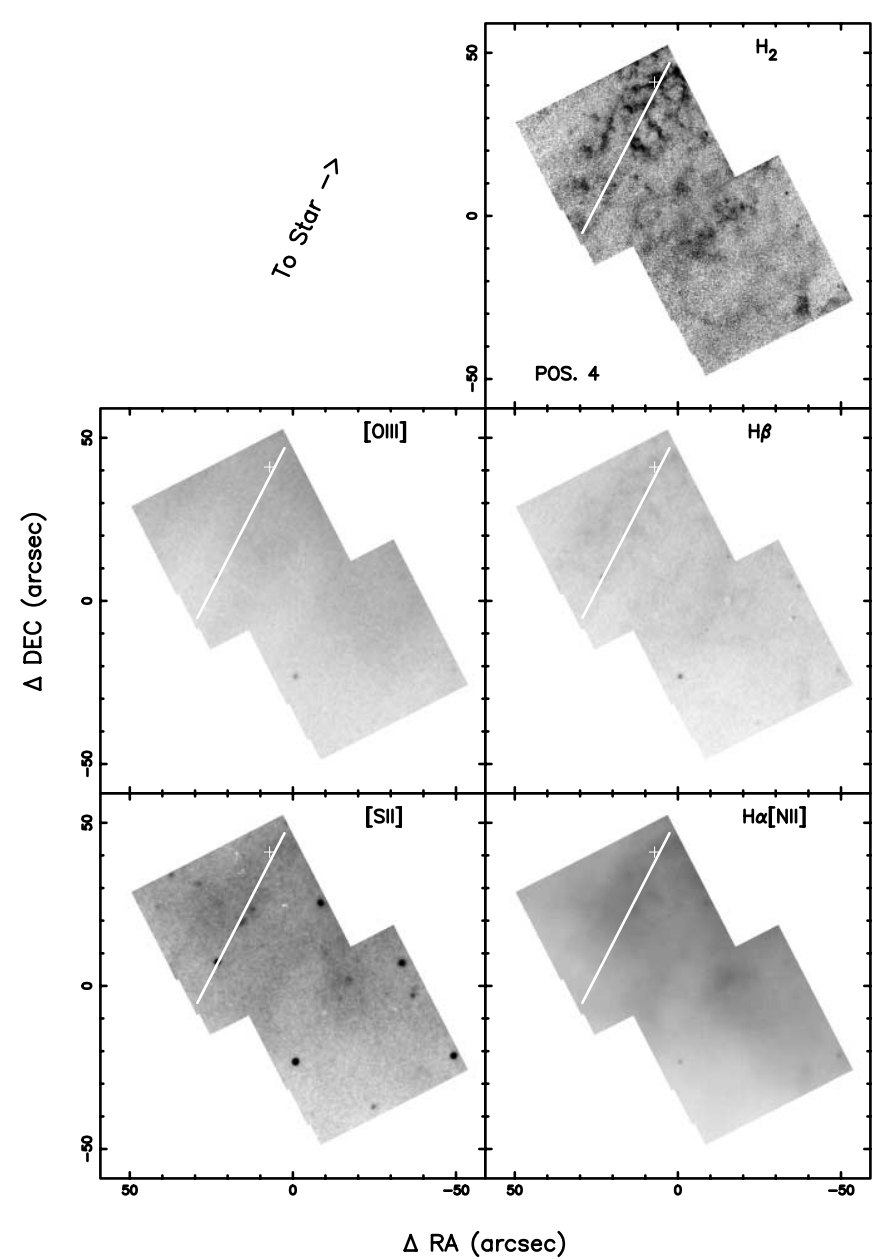

FIG. 5.-Same as Fig. 1 but for NICMOS position 4.

the photometry conversion keyword derived for the NICMOS NIC3 camera, filter F212N for the $77.1 \mathrm{~K}$ detector, appropriate for observations taken after 2002 January. Finally, to arrive at the units in Table 2 we multiplied by $212.1 \AA$, which is the filter bandwidth of the F212N filter, and divided by $2.2350443 \times$ $10^{-13} \mathrm{sr}$, which is the solid angle of the $0^{\prime \prime} 10$ pseudopixel. For each of the measured $\mathrm{H}_{2}$ knots in the NICMOS fields, we measured the surface brightness of the ionized gas emission lines, $\mathrm{H} \alpha+[\mathrm{N}$ II], [O III], $\mathrm{H} \beta$, and $[\mathrm{S}$ II] in the same way and list the results in Table 2. A conversion factor of $1.66 \times 10^{10}$ was used to convert ADUs pixel ${ }^{-1}$ to photons $\mathrm{s}^{-1} \mathrm{~cm}^{-2} \mathrm{sr}^{-1}$ for all the CTIO images, and then each image was multiplied by its photon energy $(h c) / \lambda$ to determine the surface brightness in units of ergs s$~^{-1} \mathrm{~cm}^{-2} \mathrm{sr}^{-1}$, the same as the NICMOS $\mathrm{H}_{2}$ line measurements. For the crosscuts shown in Figure 7, we applied the same conversion factors.

\section{RESULTS}

Figures 2-6 compare the NICMOS $\mathrm{H}_{2} 2.12 \mu$ m line emission images to the ionized gas tracers of [ $\left.\mathrm{O}_{\mathrm{III}}\right], \mathrm{H} \beta, \mathrm{H} \alpha+\left[\mathrm{N}_{\mathrm{II}}\right]$, and [S II] from O'Dell et al. (2004) for field positions 1-5, respectively. Our NIC3 images have better sensitivity and angular resolution than the image in Speck et al. (2002), and we observe $\mathrm{H}_{2}$ line emission at much larger distances than are seen in their large-scale, ground-based mosaic. The NICMOS field positions 1 and 2 lie closer to the central star at approximately the same projected distance. Field position 3 follows next in radial distance, with positions 5 and 4 overlapping at the farthest radial 


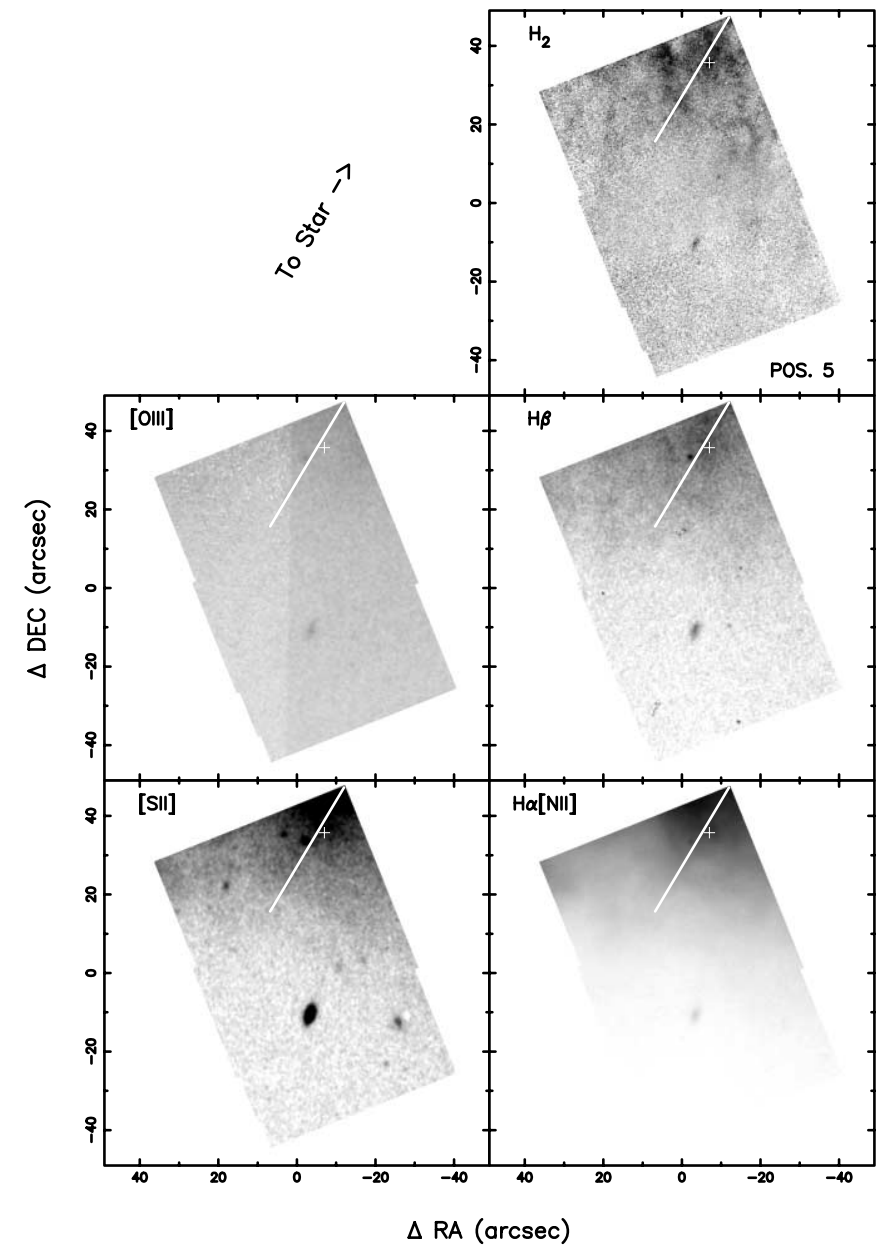

FIG. 6.- Same as Fig. 1 but for NICMOS position 5.

distances (Fig. 7). In all the NICMOS field positions the $\mathrm{H}_{2}$ emission is highly structured, revealing arcs and pillars of emission that point toward the central star (Figs. 2-6). This highly structured appearance contrasts with the smoother, less structured appearance of the ionized lines. This difference indicates that the $\mathrm{H}_{2}$ line emission is confined to the high-density neutral gas of the cometary knots (O’Dell \& Handron 1996). On the other hand, the ionized gas emission arises from both the more diffuse nebula $\left(50 \mathrm{~cm}^{-3}\right)$ and the cometary knots. Closer inspection of all five positions reveals that the $\mathrm{H} \beta$ emission structures correlate very well with the structures observed in $\mathrm{H}_{2}$. The [S II] emission appears to correlate with the $\mathrm{H}_{2}$ emission in positions 1 and 2 but does not show the structure as well as the $\mathrm{H} \beta$. This suggests that the [S II] emission is more extended, more diffuse, or less defined by the cometary knots. The $\mathrm{H} \alpha+[\mathrm{N}$ II $]$ emission, which has an appearance similar to a combination of the $\mathrm{H} \beta$ and [S II] emission, follows the $\mathrm{H}_{2}$ emission but is much more diffuse in appearance than the $\mathrm{H}_{2}$ or $\mathrm{H} \beta$.

In positions 1 and 2, the uniformity of the [O III] emission is punctuated by dark shadows of cometary knots that appear clearly in $\mathrm{H}_{2}$ emission. The $\mathrm{H}_{2}$ knots must lie in front of some highly ionized emitting gas in order to cause the extinction of the [O III $]$ emission. The fact that most of the $\mathrm{H}_{2}$ knots do not appear as [O III] shadows indicates that most of the $\mathrm{H}_{2}$ knots are confined within the neutral disk and below the highly ionized, diffuse gas apparent in $[\mathrm{O}$ III] emission. The $[\mathrm{O}$ III] emission trails off in intensity in positions 3 and 4 , having a diffuse and streaked appearance with no cometary knot emission. The [O III] emission at these more distant positions probably arises from above the disk. The bright streams of emission in the [O III] line may be examples of the crepuscular rays found in the largescale [O III] emission by O'Dell et al. (2004).

The combined crosscuts (Fig. 7) dramatically show the difference between the $\mathrm{H}_{2}$ emission and optical line ionized gas tracers. The ionized gas tracers appear to drop smoothly with increasing radius. Differences in the intensity versus radial distance for these ionized gas tracers are due to the photoionization structure of the Helix (O'Dell 1998; O'Dell et al. 2004). Of all the ionized gas tracers shown, the $\mathrm{H} \beta$ profiles reveal some clumped structure at the $5 \%-10 \%$ level on its basically smooth profile. In contrast, the $\mathrm{H}_{2}$ emission appears to fluctuate almost randomly with radial distance because of the highly clumped, knot structure of the $\mathrm{H}_{2}$ emission. The $\mathrm{H}_{2}$ intensity does not appear to decrease significantly with radial distance because the individual knots have a small range of intensities. Positions that overlap in radial distance, positions 1 and 2 , and 4 and 5, are offset in their optical line emissions because they are located at very different azimuthal positions and the nebula has a distinct variation with azimuthal angle. Interestingly, the $\mathrm{H}_{2}$ emission does not appear to have offsets in emission in the overlap regions, further supporting the possibility that the $\mathrm{H}_{2}$ emission has an almost constant level with radial distance.

Detailed comparison of the line intensities of the brighter knots in each field position also support this contrast between optical ionized gas emission and $\mathrm{H}_{2}$ emission. Table 2 lists the brightnesses of a small region in each field position, shown as a white cross in Figures 2-6. These regions were selected as bright rims of the $\mathrm{H}_{2}$ knots and hence measure the brightest $\mathrm{H}_{2}$ emission in the fields. Our NICMOS images were taken farther out in the Helix Nebula, in regions that were below the detection limit of Speck et al. (2002), $\sim 10^{-4} \mathrm{ergs} \mathrm{s}^{-1} \mathrm{~cm}^{-2} \mathrm{sr}^{-1}$, and hence complement their picture. The $\mathrm{H}_{2}$ emission from the bright $\mathrm{H}_{2}$ clumps decreases by a factor of 2 between the nearest (position 1) and most distant (position 5) clumps. In comparison, the ionized line emission drops more steeply, with the $\mathrm{H} \beta$ emission dropping by a factor of 4 and the [O III] line dropping by a factor of 5 .

TABLE 2

Surface Brightnesses of Field Positions

\begin{tabular}{|c|c|c|c|c|c|c|}
\hline Positions of $\mathrm{H}_{2}$ Knot & $\begin{array}{l}\text { Radius } \\
(\operatorname{arcsec})\end{array}$ & $\begin{array}{c}\mathrm{H}_{2} \\
\left(\operatorname{ergs~s}^{-1} \mathrm{~cm}^{-2} \mathrm{sr}^{-1}\right)\end{array}$ & $\begin{array}{c}\mathrm{H} \alpha+[\mathrm{N} \mathrm{II}] \\
\left(\operatorname{ergs~s}^{-1} \mathrm{~cm}^{-2} \mathrm{sr}^{-1}\right)\end{array}$ & $\begin{array}{c}\mathrm{H} \beta \\
\left(\operatorname{ergs~s}^{-1} \mathrm{~cm}^{-2} \mathrm{sr}^{-1}\right)\end{array}$ & $\begin{array}{c}{[\mathrm{O} \text { III] }] \lambda 5007} \\
\left(\operatorname{ergs~s}^{-1} \mathrm{~cm}^{-2} \mathrm{sr}^{-1}\right)\end{array}$ & 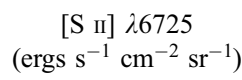 \\
\hline Position 1 & 253 & $1.27 \mathrm{E}-04$ & $7.96 \mathrm{E}-02$ & $9.33 \mathrm{E}-03$ & $4.32 \mathrm{E}-02$ & $1.43 \mathrm{E}-03$ \\
\hline Position $2 \ldots \ldots \ldots \ldots \ldots \ldots \ldots$ & 257 & $9.39 \mathrm{E}-05$ & $8.20 \mathrm{E}-02$ & $1.05 \mathrm{E}-02$ & $6.45 \mathrm{E}-02$ & $1.51 \mathrm{E}-03$ \\
\hline Position 3 & 360 & $8.55 \mathrm{E}-05$ & $4.51 \mathrm{E}-02$ & $9.30 \mathrm{E}-03$ & $9.49 \mathrm{E}-03$ & $1.09 \mathrm{E}-03$ \\
\hline 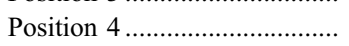 & 448 & $8.61 \mathrm{E}-05$ & $2.48 \mathrm{E}-02$ & $2.90 \mathrm{E}-03$ & $6.69 \mathrm{E}-03$ & $6.28 \mathrm{E}-04$ \\
\hline Position 5 & 436 & $5.99 \mathrm{E}-05$ & $2.42 \mathrm{E}-02$ & $2.36 \mathrm{E}-03$ & $3.44 \mathrm{E}-03$ & $6.78 \mathrm{E}-04$ \\
\hline
\end{tabular}



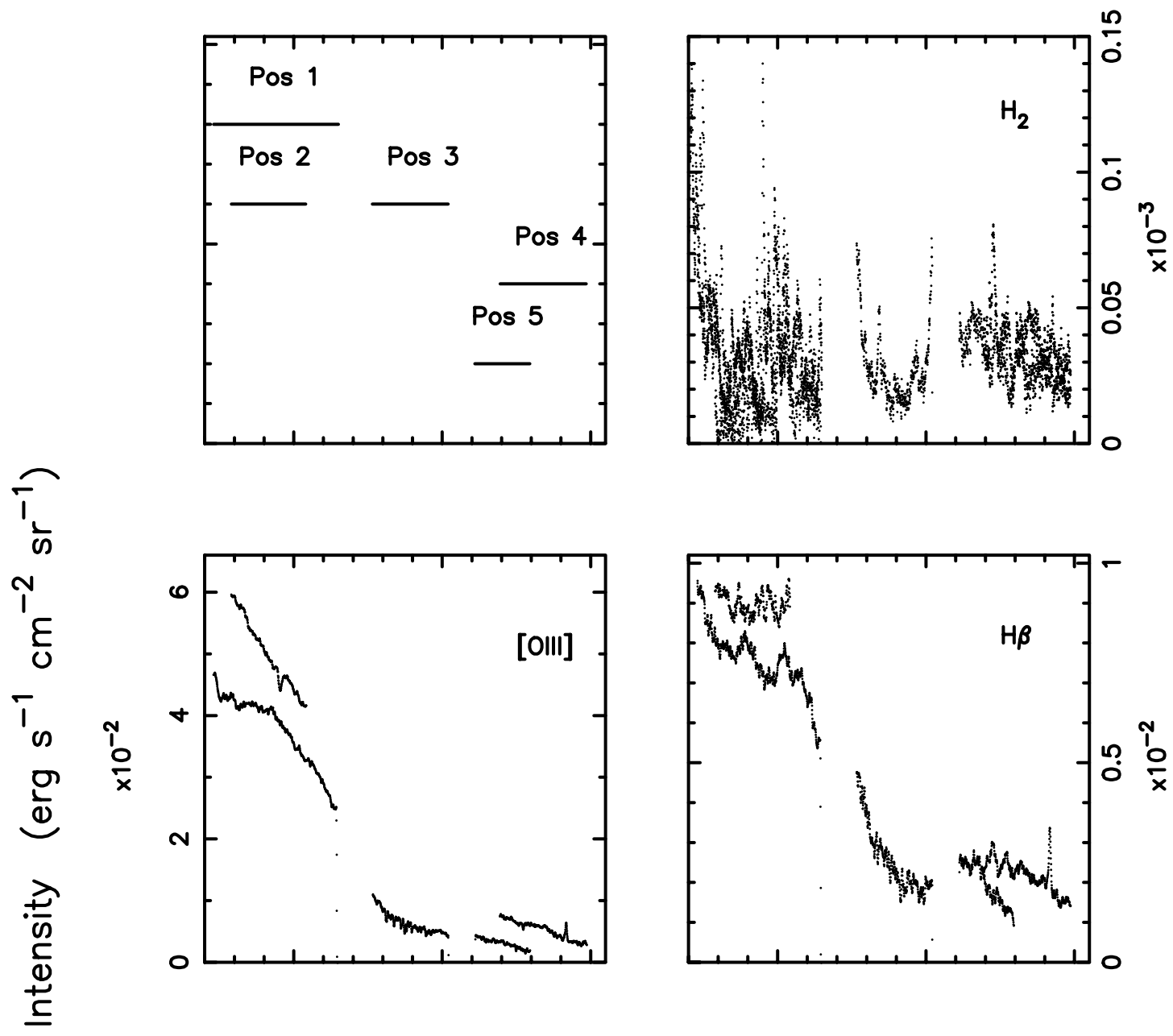

$\overbrace{\frac{1}{\omega}}^{2}$
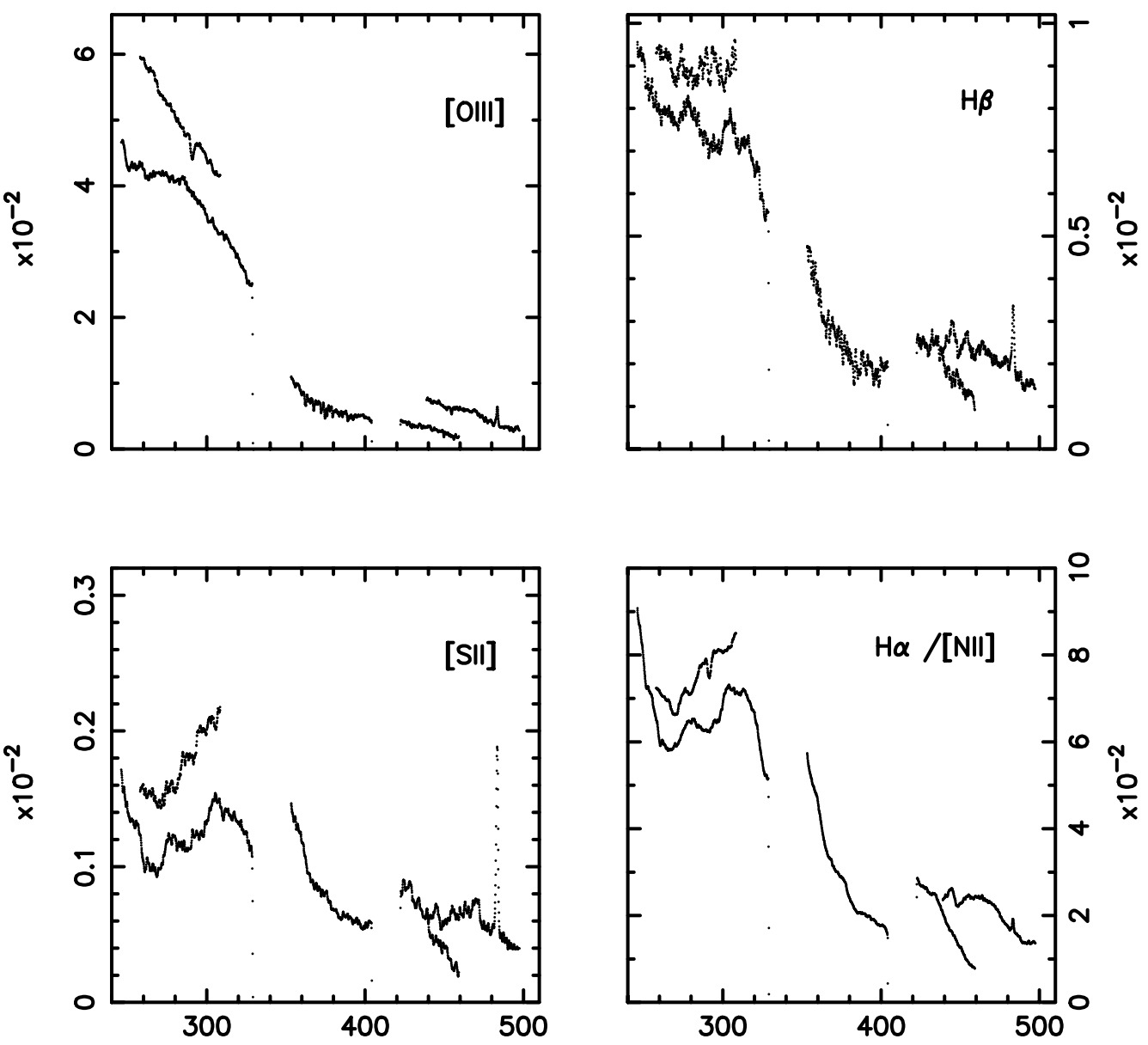

Radius from Star (")

FIG. 7.-Combined intensity radial profiles of NICMOS positions $1-5$ as a function of distance from the central star for the different emission-line tracers as labeled in the panels. The top left panel shows the horizontal location of the position slices in relative radial distances from the central star. Variations in intensity at the same radial distance but different positions are caused by the azimuthal variation of the nebular intensity emission. 


\section{DISCUSSION}

\subsection{The $\mathrm{H}_{2}$ Nebular Structure}

Speck et al. (2002) imaged the entire Helix Nebula in $\mathrm{H}_{2}$, showing that it followed the rest of the gas mass tracers, and interpreted the $\mathrm{H}_{2}$ as arising in knots in the main disk of the nebula. However, in their recent analysis of the ACS and CTIO data in combination with velocity data from the literature, O'Dell et al. (2004) revealed an entirely new three-dimensional structure of the Helix Nebula in which the main ring is broken into an inner disk and an outer ring that are almost perpendicular with respect to each other $\left(\sim 78^{\circ}\right)$. It is the superposition of these two rings that gives the Helix its helical appearance. Similar multiple-axis structures have been observed easily in edge-on polypolar PNs such as NGC 2440 (Lopez et al. 1998); however, the almost pole-on view of the Helix has made it more difficult to define its geometry. How does the full-nebula $\mathrm{H}_{2}$ image of Speck et al. (2002) fit into this new paradigm for the Helix?

Figure 8 shows the inner disk and outer ring structures discussed by O'Dell et al. (2004) superposed on the near-IR $\mathrm{H}_{2}$ image of Speck et al. (2002). The $\mathrm{H}_{2}$ near-IR emission of the inner disk is more cleanly separated from the outer ring structure than is found in the optical line tracers, which supports the conclusion that the inner disk is a separate structure from the outer ring (O'Dell et al. 2004). This cleaner separation occurs because the $\mathrm{H}_{2}$ emission arises only from the knots, and thus, there is better contrast than there is for the optical line tracers, which are diluted by the diffuse emission. The $\mathrm{H}_{2}$ emission arises at the outer edge of the inner disk, which is filled with higher excitation ionized gas in the center, as traced by [O III] (O'Dell et al. 2004). The $\mathrm{H}_{2}$ emission arising in the inner disk is also much fainter than the $\mathrm{H}_{2}$ emission in the outer ring. The opposite is true for the ionized gas tracers; they are much brighter in the inner disk than in the outer ring. This reversal suggests that the molecular gas in the inner disk has been largely photodissociated in comparison to the outer ring, and hence, the cometary knots are more evolved in the inner disk.

The outer ring does not appear very ringlike in the $\mathrm{H}_{2}$ emission but more like a ring with southern and northern arc extensions at the eastern and western edges that surround the northwestern and southeastern "plumes" mentioned by O'Dell et al. (2004). In fact, the $\mathrm{H}_{2}$ emission appears brightest in these plumes. The CO kinematics suggest that these plumes are coherent structures (Young et al. 1999). One possible explanation is that the outer ring defined by O'Dell et al. (2004) is really part of an outer bipolar structure, and the southern and northern arcs are the limb-brightened edges of the bicones in the outer structure. Such an interpretation is in line with the Helix Nebula being a polypolar PN viewed pole-on. The edge-on polypolar PN NGC 2440 has an inner torus of $\mathrm{H}_{2}$ emission and an outer bipolar nebula of $\mathrm{H}_{2}$ emission with axes of symmetry that are tilted with respect to one another (Latter et al. 1995).

Our NICMOS field positions appear to lie in some of the fainter or nonexistent regions of $\mathrm{H}_{2}$ emission as observed by Speck et al. (2002), whose observations had less sensitivity than our study. Positions 1 and 2 are located outside the southern edge of the inner disk in what appears to be a gap between the inner disk and the outer structures. Position 3 lies to the southwest of the outer structure, where no apparent $\mathrm{H}_{2}$ emission appears in the $\mathrm{H}_{2}$ image by Speck et al. (2002). Positions 4 and 5 are located even farther away from previously detectable $\mathrm{H}_{2}$ emission south of the nebula. Positions 3, 4, and 5 also lie in regions in which no $\mathrm{H}$ i $21 \mathrm{~cm}$ line emission was detected by Rodríguez et al. (2002) and no CO emission was detected by

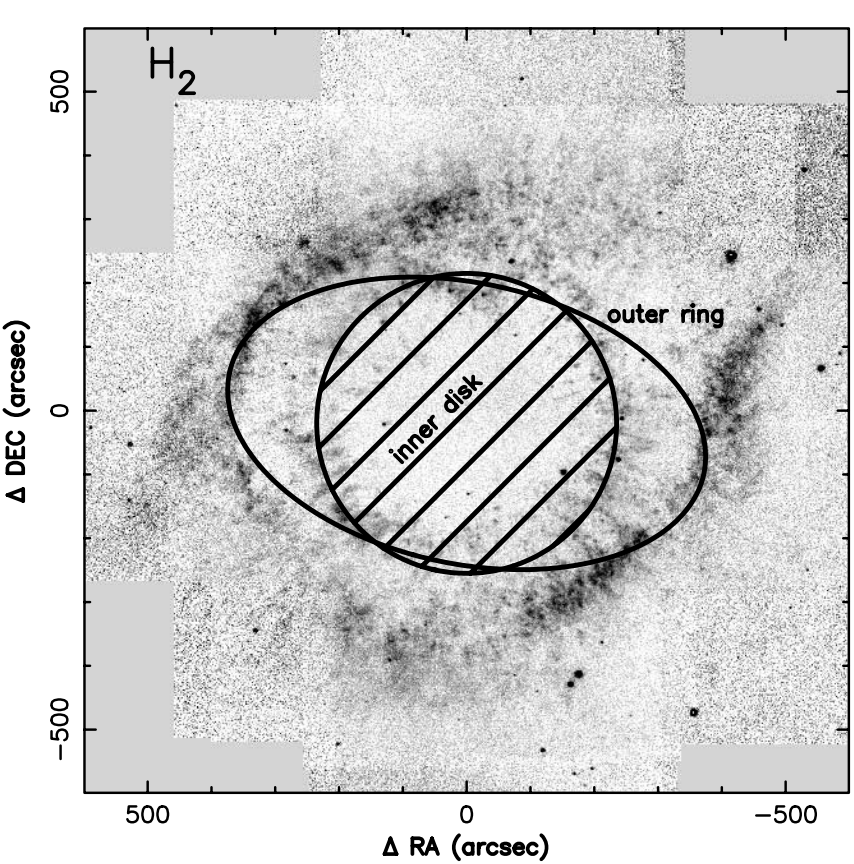

FIG. 8.- $\mathrm{H}_{2}$ line emission image from Speck et al. (2002), with the inner disk and outer ring structures labeled as identified by O'Dell et al. (2004).

Young et al. (1999). The number density of $\mathrm{H}_{2}$ knots and the area filling factor of $\mathrm{H}_{2}$ emission decreases with increasing radius and is lowest in positions 3, 4, and 5 (Figs. 4-6). In the large beams of these radio line observations $\left(\sim 31^{\prime \prime}\right.$ FWHM for $\mathrm{CO}$ and $\sim 42^{\prime \prime} \mathrm{FWHM}$ for $\mathrm{H}_{\mathrm{I}} 21 \mathrm{~cm}$ ), the intensity of the neutral gas emission from these knots is beam-diluted and falls below the detection limit of the radio observations.

\subsection{The Multitude of Molecular Knots in the Helix}

Our observations clearly show that the morphology of the molecular hydrogen emission is highly clumped in comparison to the ionized gas tracers. Figure 9 shows a multicolor image comparing the [O $\mathrm{OII}]$, the $\mathrm{H} \alpha+\left[\mathrm{N}\right.$ II] , and the $\mathrm{H}_{2}$ emission for position 1 . The structure observed in this image is primarily due to the $\mathrm{H}_{2}$ emission clumps. In fact, the $\mathrm{H}_{2}$ emission images are striking by their lack of diffuse $\mathrm{H}_{2}$ emission. Close inspection of the more intense regions shows they are composed of overlapping knots of $\mathrm{H}_{2}$ emission. Hence, we confirm the conclusion of Speck et al. (2002) that the molecular hydrogen is confined to the high-density knots such as is seen in the optical by O'Dell \& Handron (1996). A similar conclusion was reached by Speck et al. (2003) for the Ring Nebula based on a comparison of highresolution ground-based $\mathrm{H}_{2}$ emission images to the optical HST images. Thus, in two evolved PNs, the Helix and the Ring Nebulae, the $\mathrm{H}_{2}$ line emission is highly structured and confined to knots.

The near-IR $\mathrm{H}_{2}$ emission provides us with the highest angular resolution map of the neutral gas knots in the Helix Nebula. Previous work has suggested that the knots contain all the neutral gas detected at substantially lower angular resolution in CO emission ( 31" FWHM) by Young et al. (1999), C I emission $\left(\sim 15^{\prime \prime}\right.$ FWHM) by Young et al. (1997), H I emission ( $\sim 2^{\prime \prime}$ FWHM) by Rodríguez et al. (2002), and $\mathrm{H}_{2}$ line emission ( $4^{\prime \prime}$ FWHM) by Speck et al. (2002). However, all previous neutral gas studies have had insufficient resolution and sensitivity to separate and determine the structure and number density of these neutral gas knots. 


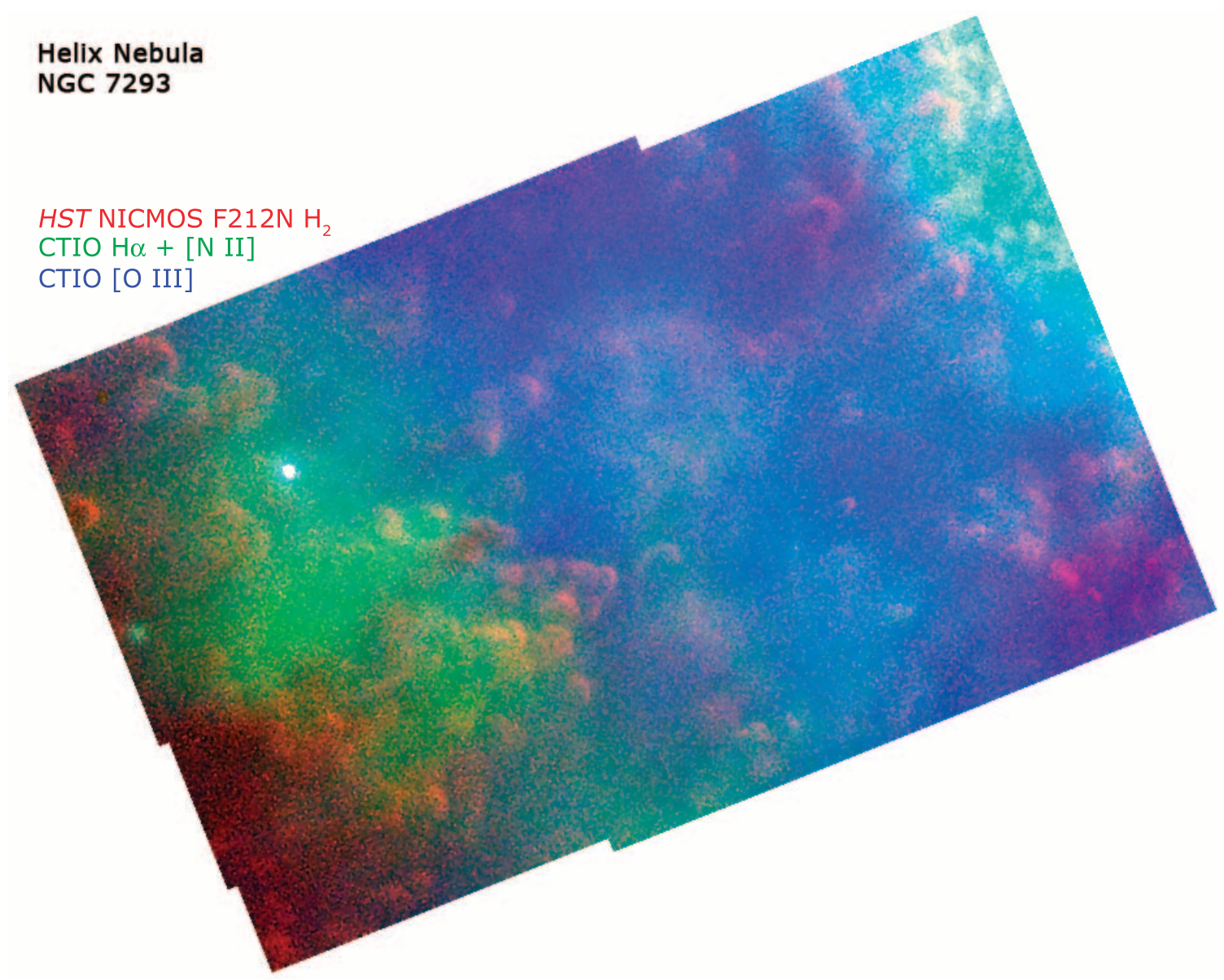

FIG. 9.-NICMOS position 1 in three colors representing the $\mathrm{H}_{2}$, the $\mathrm{H} \alpha+[\mathrm{N} \mathrm{II}]$, and the [O III] emission. This color comparison reemphasizes the clumpy nature of $\mathrm{H}_{2}$ line emission in comparison to the more diffuse ionized gas line emission.

The optical study of the knots by O’Dell \& Handron (1996) provided an initial lower limit for the total number of cometary knots of 3500 in the entire nebula. They base this estimate on the extrapolation of the number density of knots they can identify in their WFPC2 optical images versus the distance from the center of the nebula. Our $\mathrm{H}_{2}$ images reveal that many more molecular knots exist as defined by the $\mathrm{H}_{2}$ emission arcs than can be identified in the optical images (Figs. 2-6). For example, in the NICMOS field position 1 the number of knots that appear as [O III] shadows is less than 10; however, the number of arcshaped $\mathrm{H}_{2}$ emission structures is $\sim 150$. In Table 3 we list the number of knots, which we identify by arcs of $\mathrm{H}_{2}$ emission, and the FOV of the image. The number density of knots is simply the total divided by the FOV. The area filling factor of $\mathrm{H}_{2}$ emission is the percentage of the FOV that contains $\mathrm{H}_{2}$ emission structures above a $1 \sigma$ threshold intensity $\left[\sim(1-2) \times 10^{-5} \mathrm{ergs} \mathrm{s}^{-1}\right.$ $\left.\mathrm{cm}^{-2} \mathrm{sr}^{-1}\right]$. The total number of knots, the number density of knots, and the area filling factors are highest for positions 1 and 2 and decrease with larger radial distance from the star, as seen in positions 4 and 5. Interestingly, the peak surface brightness of the knots does not decrease significantly with radial distance, as we see in Figure 7 and Table 2.

We estimate the total number of molecular knots in the Helix by scaling the number of knots we observe in our NICMOS images to the total angular size of the Helix. If we look at the $\mathrm{H}_{2}$ image of Speck et al. (2002) we find that our NICMOS field positions 1 and 2 land in a region of average or slightly below average $\mathrm{H}_{2}$ intensity. So we make a conservative estimate of the total number of knots by using the average knot number density of positions 1 and 2, 0.041 knots $\operatorname{arcsec}^{-2}$. The $\mathrm{H}_{2}$ emission region is an annulus with an inner radius of $\sim 170^{\prime \prime}$ and an outer radius of $450^{\prime \prime}$, covering a total angular area of $5.5 \times 10^{5} \operatorname{arcsec}^{2}$. Multiplying the average knot number density by the total angular area equals $\sim 23,000$ molecular hydrogen knots in the Helix

TABLE 3

Number of $\mathrm{H}_{2}$ Knots

\begin{tabular}{|c|c|c|c|c|}
\hline Positions & Total Number of Knots & $\begin{array}{l}\text { FOV Area } \\
(\operatorname{arcsec})\end{array}$ & $\begin{array}{l}\text { Number Density } \\
\left(\text { knots } \operatorname{arcmin}^{-2}\right)\end{array}$ & $\begin{array}{c}\text { Area Filling Factor of } \mathrm{H}_{2} \text { Emission } \\
(\%)\end{array}$ \\
\hline Position 1 .......................... & 150 & $50 \times 80$ & 135 & 80 \\
\hline Position $2 \ldots \ldots \ldots \ldots \ldots$ & 180 & $50 \times 80$ & 162 & 70 \\
\hline Position 3 & 90 & $50 \times 80$ & 81 & 60 \\
\hline Position 4 .......................... & 90 & $50 \times 100$ & 65 & 50 \\
\hline Position 5 ......................... & 20 & $50 \times 80$ & 18 & 25 \\
\hline
\end{tabular}




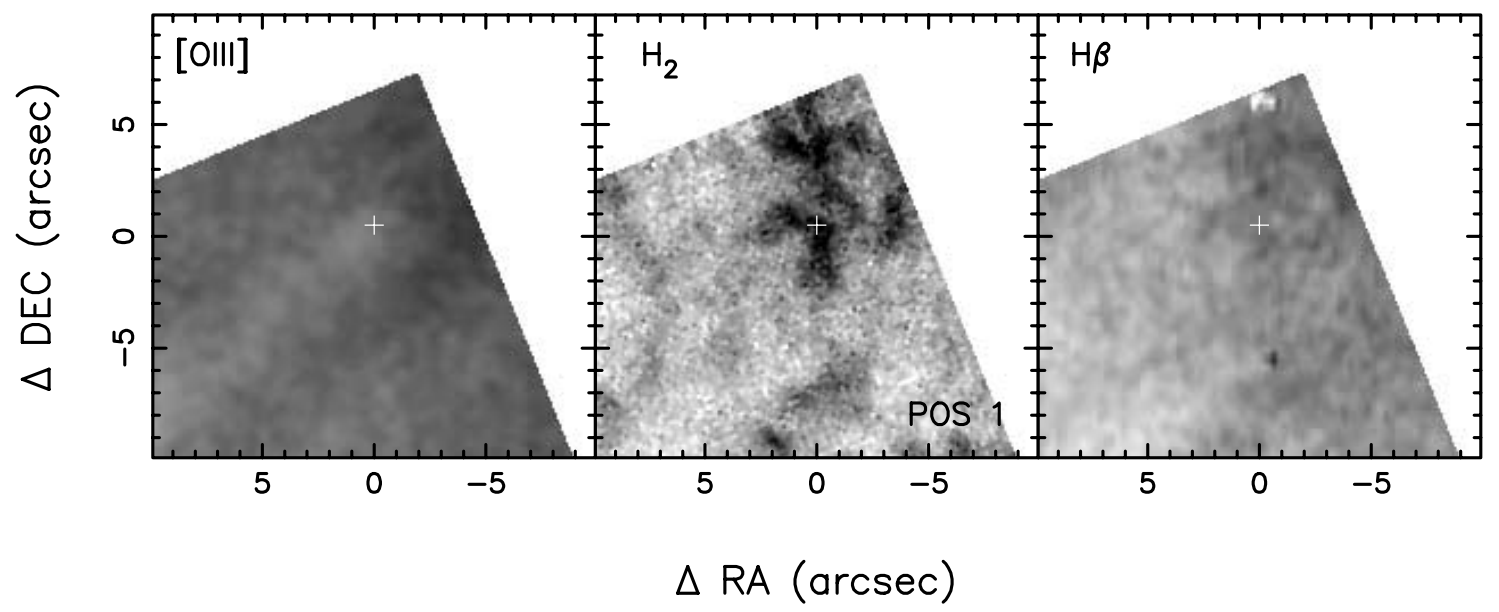

FIG. 10.- Selected $\mathrm{H}_{2}$ knot from NICMOS position 1 shown in [O III] (shadow), $\mathrm{H}_{2}$, and $\mathrm{H} \beta$ line emission. The white cross, which marks the location of the line emission measurement, is a guide for the relative position of these emissions. The $\mathrm{H}_{2}$ and $\mathrm{H} \beta$ line emissions appear to be spatially coincident, while the [O III] shadow column ends at the cross and the $\mathrm{H}_{2}$ arc emission structure.

Nebula, or a factor of 6.5 greater than previous estimates based on optical images (O’Dell \& Handron 1996). The estimated mass of a single Helix knot is $\sim 1.5 \times 10^{-5} M_{\odot}$ (O'Dell \& Handron 1996) or $10^{-4} M_{\odot}$ (Young et al. 1997). However, the Young et al. (1997) C I study defined a "knot" to be $30^{\prime \prime} \times 10^{\prime \prime}$ in size, which would include $\sim 10 \mathrm{H}_{2}$ knots if we assume the knot densities of position 1. Because our $\mathrm{H}_{2}$ knots appear similar in shape and size to the optical knots observed by O'Dell \& Handron (1996), we adopt their mass estimate for individual knots. If, for simplicity, we assume that all $\mathrm{H}_{2}$ knots have similar properties with an average mass of $\sim 1.5 \times 10^{-5} M_{\odot}$ (O'Dell \& Handron 1996), then the total neutral gas mass of the Helix Nebula is $\sim 0.35 M_{\odot}$. Our neutral gas mass estimate is substantially larger than the $0.01 M_{\odot}$ estimated by O'Dell \& Handron (1996), who underestimated the total number of knots. It is also higher than the $0.18 M_{\odot}$ estimated from the $\mathrm{CO}$ observations that were corrected for the atomic $\mathrm{C}_{\mathrm{I}}-\mathrm{emitting}$ gas by Young et al. (1999), who have underestimated the mass because the $\mathrm{CO}$ observations do not detect all the molecular gas. Our estimate of the neutral gas mass is comparable to the ionized gas mass of $0.3 M_{\odot}$ (Henry et al. 1999), and the total gas mass in the nebula is $>0.65 M_{\odot}$ in the main part of the disk. The mass-loss rate, which created the main disk over $\sim 28,000 \mathrm{yr}$, is $\dot{M}>2.3 \times 10^{-5} M_{\odot} \mathrm{yr}^{-1}$. Such a large mass-loss rate supports the independent conclusion that the Helix's progenitor star was massive, $6.5 M_{\odot}$, resulting in a present-day core mass of $0.93 M_{\odot}$ (Gorny et al. 1997).

\subsection{The Evolution of $\mathrm{H}_{2}$ Knots}

Figure 10 shows a close up of the bright knot in NICMOS field position 1 in three tracers, [O III], $\mathrm{H}_{2}$, and $\mathrm{H} \beta$. The knot extinguishes the $[\mathrm{O}$ III] emission, and its long shadow runs off to the bottom left corner. The arc-shaped head of the knot is clearly observed in the $\mathrm{H}_{2}$ emission and is apparent, at lower contrast, in the $\mathrm{H} \beta$ emission. Close comparison of the molecular hydrogen emission shows that it almost coincides with the $\mathrm{H} \beta$ and is displaced toward the star from the shadowed regions of the [O III] absorption of the knot (Fig. 10). This structure suggests that the $\mathrm{H}_{2}$ emission arises in mini-PDRs on the clump surfaces that point toward the central star. The intensities measured for the $\mathrm{H}_{2}$ emission are consistent with the PN-PDR models of Natta \& Hollenbach (1998). Comparison of this knot structure (Fig. 10) with the detailed study of an optically bright knot in the inner- most regions of the Helix (Huggins et al. 2002) reveals a change in the knot's structure. The most inward knot studied in $\mathrm{H}_{2}$ by Huggins et al. (2002) is pillar-like, with a well developed crown structure. The $\mathrm{H} \alpha$ emission in this knot lies closer to the central star compared to the $\mathrm{H}_{2}$ emission, and the $\mathrm{H}_{2}$ emission lies closer to the central star than the $\mathrm{CO}$ emission of the knot. This stratified structure is what we expect for a PDR. However, we do not see a separated stratification of the $\mathrm{H}_{2}$ and $\mathrm{H} \beta$ emission from our example knot from position 1. This difference in layered versus nonlayered structure of the knots' PDRs suggests that the innermost knot has a more evolved PDR front than the position 1 knot.

A comparison of the different field positions shows a progression in the morphology of the $\mathrm{H}_{2}$ knots with radial distance from the central star. The positions closest to the central star (positions 1 and 2) have numerous knots, with cometary structures, i.e., arcs at the top spires. The farthest positions (4 and 5) have substantially less knots and less structure to the knots. Position 3, which lies in between the two extremes, has a middle density of knots that are lined in continuous rows with fewer spire structures. A similar trend from highly structured to almost amorphous was observed in the $\mathrm{H} \alpha$ structure of the knots by O'Dell et al. (2004). The $\mathrm{H}_{2}$ emission traces this morphological change to larger radial distances from the central star. At the outer edge of the nebula, the $\mathrm{H}_{2}$ knots are the least structured. This progression supports the idea that the initial stages of the structure formation are caused by instabilities in the interacting winds' front or the ionization front that are later refined by the photoexcitation.

\subsection{The $\mathrm{H}_{2}$ Knots as Mini-PDRs}

When molecular hydrogen was first imaged in PNs it was found that the brightness was too high for the molecular emission to have originated in PDRs and thus be attributed to shock excitation (Beckwith et al. 1978; Zuckerman \& Gatley 1988). However, the PDR models used for these comparisons were designed for interstellar molecular clouds rather than circumstellar nebulae around rapidly evolving stars. As such, these models only included farUV photons and failed to include the soft X-ray emission inherent to very hot $(>100,000 \mathrm{~K})$ white dwarfs. The temperature of the white dwarf also changes as it evolves during the lifetime of the PN. Furthermore, the original models assumed that the cloud was homogeneous, while the gas around PNs is clearly 
very clumpy in structure. In addition, the interstellar clouds in these models are not expanding, whereas this is the case for the gas around PNs, causing a change in the optical depth of the gas and therefore allowing the photons to penetrate the gas more easily. The models of Natta \& Hollenbach (1998) included three of these factors (evolving central star, expanding gas, and X-ray photons) into their PDR model for PNs and showed that the molecular hydrogen emission was approximately consistent with excitation of $\mathrm{H}_{2}$ in PDRs in these environments. The Natta \& Hollenbach (1998) model has been applied to three PNs, NGC 2346 (Vicini et al. 1999), the Helix Nebula (Speck et al. 2002), and the Ring Nebula (Speck et al. 2003).

Our new observations of the Helix molecular knots confirm that the PDR gas in the Helix resides in a multitude of miniPDRs, not in a diffuse molecular component (Speck et al. 2002; Huggins et al. 2002). The other, perhaps more surprising, fact is that the intensity of individual knots remains fairly constant with distance from the central star in the range $(5-9) \times 10^{-5} \mathrm{ergs}$ $\mathrm{s}^{-1} \mathrm{~cm}^{-2} \mathrm{sr}^{-1}$ (Table 2; Fig. 7). The apparent, almost random variation in the $\mathrm{H}_{2}$ line intensity with respect to radius (Fig. 7) occurs because the number density of knots varies along the crosscut, not the intensity of the knots themselves. The relative consistency of the $\mathrm{H}_{2}$ line intensity of the individual knots with respect to distance from the central star further supports the belief that the PDRs are distributed as mini-PDRs, and there is not one PDR front for the Helix but a multitude of them. The higher rotational lines of $\mathrm{H}_{2}$, which were observed with ISOCAM by Cox et al. (1998), indicate a thermalized temperature of the gas of $\sim 900 \mathrm{~K}$, which appears to be independent of distance from the central star. The gas density within the knots, $\sim 10^{4}-10^{5} \mathrm{~cm}^{-3}$, is high enough to thermalize the flourescently excited $\mathrm{H}_{2}$ gas. The observed $\mathrm{H}_{2}$ line intensities of the Helix and the derived molecular gas temperature are approximately consistent with the PN/PDR theory of Natta \& Hollenbach (1998). For the evolution of the most massive stars, e.g., core mass $0.836 M_{\odot}$ and at the age of the Helix, $\sim 16,000 \mathrm{yr}$, Natta \& Hollenbach (1998) predict $\mathrm{H}_{2}$ gas temperatures of $\sim 1000 \mathrm{~K}$ and $\mathrm{H}_{2} 2.12 \mu \mathrm{m}$ line intensities of $\sim 5 \times 10^{-5} \mathrm{ergs} \mathrm{s}^{-1} \mathrm{~cm}^{-2} \mathrm{sr}^{-1}$, in good agreement with the observed temperature and the individual $\mathrm{H}_{2}$ line intensities of the majority of knots. At this stage of the PN/PDR evolution, the $\mathrm{H}_{2}$ line intensity decreases only gradually with time, and the heating of the molecular gas is dominated by the soft X-ray emission of its $123,000 \mathrm{~K}$ central star (Bohlin et al. 1982).

Despite this approximate success of the PN/PDR models of Natta \& Hollenbach (1998), these models fall short of complete success. The brightest $\mathrm{H}_{2}$ intensity is almost a factor of 10 larger than the Natta \& Hollenbach (1998) prediction. Recent model calculations of the $\mathrm{H}_{2}$ intensity of knots in radiative equilibrium with the stellar radiation field by O'Dell et al. (2005) also underpredict the $\mathrm{H}_{2}$ line intensities. The solution to this underprediction of both models may be to combine the time evolution aspects of the Natta \& Hollenbach (1998) models with the inclusion of knots, as modeled by O'Dell et al. (2005). For example, the time-dependent process of photoevaporation causes an advection of $\mathrm{H}_{2}$ from the surface of the knot and a propagation of the PDR front into the knot, which may boost the $\mathrm{H}_{2}$ emission because of the constant photodissociation of fresh molecular gas (Natta \& Hollenbach 1998). Evidence for photoevaporation has been found in the ionized gas studies of the innermost knots (O'Dell et al. 2000), which are knots that are directly exposed to the central star light. Thus, the higher $\mathrm{H}_{2}$ knots could be those that are directly exposed to the central starlight and experiencing photoevaporation. However, most knots experience a softer starlight that has been attenuated by intervening knots of molecular gas and dust. Also, the brightest $\mathrm{H}_{2}$ intensity detected by Speck et al. (2002), $\sim 3 \times 10^{-4}$ ergs s${ }^{-1} \mathrm{~cm}^{-2} \mathrm{sr}^{-1}$, may be the result of multiple knots along the line of sight, i.e., filling factors greater than 1 that have a multiplying effect on the intensity. This raises an interesting point that the spatial distribution of $\mathrm{H}_{2}$ over the entire nebula varies, not because of substantial $\mathrm{H}_{2}$ intensity variation, as one might expect in a PDR front, but rather because the apparent $\mathrm{H}_{2}$ surface brightness is proportional to the number density of $\mathrm{H}_{2}$ knots.

\section{CONCLUSIONS}

New observations of the $\mathrm{H}_{2} 2.12 \mu \mathrm{m}$ line reveal several new aspects to the molecular knots of the Helix Nebula. The $\mathrm{H}_{2}$ images reveal that the knots have arcuate structures with the apex pointing toward the central star. These molecular hydrogen knots are most highly structured in the field positions closest to the central star and become increasingly less structured with increasing radius. All the $\mathrm{H}_{2}$ emission is confined to knots. In contrast, the ionized gas tracers have a significant component of diffuse ionized gas emission. Using the number density of molecular hydrogen knots in the five NICMOS field positions, we estimate the total number of knots to be $\sim 23,000$, a factor of 6.5 more than previous estimates based on optical images. The total neutral gas mass in the Helix based on these new knot estimates is $0.35 M_{\odot}$ assuming an average mass of $\sim 1.5 \times 10^{-5} M_{\odot}$ for the individual knots based on previous work by O'Dell \& Handron (1996). The $\mathrm{H}_{2}$ emission structure of the entire Helix Nebula supports the recent interpretation of the Helix as a nearly pole-on polypolar PN. The average intensity of (5-9) $\times 10^{-5}$ ergs s $\mathrm{sm}^{-1} \mathrm{sr}^{-1}$ remains relatively constant with projected distance from the central star. The temperature and $\mathrm{H}_{2} 2.12 \mu \mathrm{m}$ intensity of the knots suggest an origin in the photodissociation regions of the individual knots; however, theoretical models for the PDRs in PNs do not adequately reproduce the $\mathrm{H}_{2}$ intensity. The brightest knots appear in regions of more numerous knots and may be exposed to direct starlight that may cause rapid photoevaporation in comparison to the more embedded knots of the disk.

We gratefully acknowledge the work of the many STScI colleagues who contributed to the observational setup of this project. We thank Zoltan Levay, who superposed the NICMOS fields on the combined CTIO and ACS image. This work was supported in part by STScI grant GO 01041 and by internal STScI funds DDRF D0001.82319.
Beckwith, S., Gatley, I., \& Persson, S. E. 1978, ApJ, 219, L33

Bohlin, R. C., Harrington, J. P., \& Stecher, T. P. 1982, ApJ, 252, 635

Burkert, A., \& O’Dell, C. R. 1998, ApJ, 503, 792

Capriotti, E. R. 1973, ApJ, 179, 495

Cox, P., et al. 1998, ApJ, 495, L23

Gonçalves, D. R., Corradi, R. L. M., \& Mampaso, A. 2001, ApJ, 547, 302

Gorny, S. K., Stasinska, G., \& Tylenda, R. 1997, A\&A, 318, 256

\section{REFERENCES}

Harris, H. C., Dahn, C. C., Monet, D. G., \& Pier, J. R. 1997, in IAU Symp. 180, Planetary Nebulae, ed. H. J. Habing \& H. J. G. L. M. Lamers (Dordrecht: Kluwer), 40

Henry, R. B. C., Kwitter, K. B., \& Dufour, R. J. 1999, ApJ, 517, 782

Huggins, P. J., Forveille, T., Bachiller, R., Cox, P., Ageorges, N., \& Walsh, J. R. 2002, ApJ, 573, L55

Huggins, P. J., \& Healy, A. P. 1986, ApJ, 305, L29 
Kastner, J. H., Gatley, I., Merrill, K. M., Probst, R., \& Weintraub, D. 1994, ApJ, 421, 600

Latter, W. B., Kelly, D. M., Hora, J. L., \& Deutsch, L. K. 1995, ApJS, 100, 159

Lopez, J. A., Meaburn, J., Bryce, M., \& Holloway, A. J. 1998, ApJ, 493, 803

McCullough, P. R., et al. 2002, BAAS, 201, 143.01

Meaburn, J., Walsh, J. R., Clegg, R. E. S., Walton, N. A., Taylor, D., \& Berry, D. S. 1992, MNRAS, 255, 177

Meixner, M., McCullough, P., Hartman, J., O’Dell, R., \& Speck, A. K. 2004, in ASP Conf. Ser. 313, Asymmetrical Planetary Nebulae III: Winds, Structure, and the Thunderbird, ed. M. Meixner et al. (San Francisco: ASP), 234

Natta, A., \& Hollenbach, D. 1998, A\&A, 337, 517

O’Dell, C. R. 1998, AJ, 116, 1346

O’Dell, C. R., Balick, B., Hajian, A. R., Henney, W. J., \& Burkert, A. 2002, AJ, 123,3329

O’Dell, C. R., \& Handron, K. D. 1996, AJ, 111, 1630

O’Dell, C. R., Henney, W. J., \& Burkert, A. 2000, AJ, 119, 2910
O’Dell, C. R., Henney, W. J., \& Ferland, G. J 2005, AJ, 130, 1720

O’Dell, C. R., McCullough, P. R., \& Meixner, M. 2004, AJ, 128, 2339

Rodríguez, L. F., Goss, W. M., \& Williams, R. 2002, ApJ, 574, 179

Speck, A. K., Meixner, M., Fong, D., McCullough, P. R., Moser, D. E., \& Ueta, T. 2002, AJ, 123, 346

Speck, A. K., Meixner, M., Jacoby, G. H., \& Knezek, P. M. 2003, PASP, 115, 170

Thompson, R. I., Rieke, M., Schneider, G., Hines, D. C., \& Corbin, M. R. 1998, ApJ, 492, L95

Vicini, B., Natta, A., Marconi, A., Testi, L., Hollenbach, D., \& Draine, B. T. 1999, A\&A, 342, 823

Young, K., Cox, P., Huggins, P. J., Forveille, T., \& Bachiller, R. 1997, ApJ, 482, L101

-1999, ApJ, 522, 387

Zuckerman, B., \& Gatley, I. 1988, ApJ, 324, 501 